\title{
Collective Luminescence and Phonon-Induced Processes in Double Quantum Dots
}

\author{
P. Machnikowski ${ }^{a, *}$, K. RoszAK ${ }^{a, b}$ And A. SiteK ${ }^{a, c}$ \\ ${ }^{a}$ Institute of Physics, Wrocław University of Technology, Wybrzeże Wyspiańskiego 27, 50-370 Wrocław, Poland \\ ${ }^{b}$ Department of Condensed Matter Physics, Faculty of Mathematics and Physics, Charles University \\ 12116 Prague, Czech Republic \\ ${ }^{c}$ Institut für Theoretische Physik, Nichtlineare Optik und Quantenelektronik \\ Technische Universität Berlin, 10623 Berlin, Germany
}

\begin{abstract}
We study the evolution of a quantum state of a double quantum dot system interacting with the electromagnetic environment and with the lattice modes, in the presence of a coupling between the two dots. We propose a unified approach to the simulation of the system evolution under joint impact of the two reservoirs. We discuss the sub- and superradiant radiative decay of the system, the phonon-induced decay of entanglement between the dots, and the transfer of excitation between them.
\end{abstract}

PACS numbers: 03.65.Yz, 78.67.Hc, 03.67.Lx, 71.38.-k, 73.63.Kv

\section{Introduction}

Semiconductor structures composed of two closely spaced quantum dots (QDs) have attracted enormous attention in recent years. Pairs of vertically stacked QDs with spatial separation down to single nanometers can be obtained in a two-layer self-assembled growth process, where the strain distribution favors QD nucleation in the second layer on top of the QDs formed in the first layer $[1,2]$. The development of the manufacturing technologies $[3,4]$ has made it possible to achieve structures built of nearly identical dots, with the splitting of ground state transition energies down to single $\mathrm{meV}$ (very likely interaction-limited) $[5,6]$. The state space of such a double quantum dot (DQD) is obviously richer than that of a single QD $[7,8]$ and allows, e.g., for entanglement between the dots. Also the recombination and relaxation processes in DQDs show many features which cannot appear in individual QDs. The quantum coherence of carrier states in DQDs are affected by the interference and collective effects that appear in the interaction of such systems with their radiative environment (electromagnetic vacuum) and with the surrounding crystal lattice (phonons).

Optical properties of DQDs may be strongly modified due to collective interaction of sufficiently closely spaced QDs with the electromagnetic (EM) field. These collective effects have been extensively studied for atomic systems [9] where they manifest themselves by superra-

* corresponding author; e-mail: pawel.machnikowski@pwr.wroc.pl diant emission [10]. A signature of superradiant behavior was also observed in ensembles of QDs [11]. On the other hand, the collective interaction leads to the appearance of subradiant states which are decoupled from the environment and, therefore, do not undergo decoherence. It has been proposed to use these states for noiseless encoding of quantum information $[12,13]$.

Another area where new features appear for DQD systems is the decoherence due to carrier-phonon coupling. Dephasing of carrier states in QDs due to carrier-phonon dynamics has been observed experimentally as a decay of nonlinear optical response in a four-wave mixing experiment with ultrashort pulses [14, 15]. A characteristic feature of the phonon-induced dephasing in QDs is that it is only partial, i.e., after a few picoseconds of carrierphonon dynamics, the degree of coherence (i.e., the values of the off-diagonal elements of the density matrix) reaches a certain finite level, depending on the system geometry and temperature $[16,17]$. In DQDs and regular QD arrays, the degree of dephasing may be reduced by encoding the logical qubit values into many-exciton states over a QD array [18]. Phonon-induced dephasing is also detrimental to entanglement in DQDs and larger QD arrays. The impact of the partial dephasing on the entanglement is very strong since the latter is more prone to dephasing than local coherence and may be completely destroyed even though the decoherence is only partial [19-21]. Moreover, because of the delicate aspect of inter-subsystem coherences involved, the decay of entanglement due to dephasing strongly depends on the nature of environmental interaction (the same vs. different reservoirs for the two subsystems) [22]. 
Various types of coupling between the dots change the properties of the system even further. Theoretical calculations show that for closely spaced dots, tunnel coupling (wave function overlap) between the dots should strongly affect their electronic structure [23-25]. Optical spectra of such structures indeed show clear manifestations of electronic coupling [6, 26-29].

On the other hand, wave function overlap is not the only mechanism of interaction between the QDs. In fact, for QD separations of about $10 \mathrm{~nm}$ the energetically lowest states (in the absence of external fields) correspond to spatially direct excitons localized in individual QDs [25]. Such states are still bound by the Coulomb interaction. While the static ("direct") dipole coupling preserves the occupations of the individual QDs, the Förster interaction via interband dipole moments [30, 31] (first introduced in the context of molecular systems [32,33]) makes it possible to transfer the exciton occupation between the dots. In contrast to the tunnel coupling, which is analogous to chemical bonding between atoms and, therefore, turns the two dots into one quantum system (a quantum dot molecule), the dipole couplings are rather like van der Waals forces between separate entities. Therefore, QDs coupled by this kind of interaction are much closer to the general paradigm of well-defined, separate qubits [34] on which, however, collective quantum operations (multi-qubit gates) can be performed, based on the interactions between the systems.

In a closed system the (usually very weak) Förster interaction has considerable effects only very close to resonance $[30,31]$. However, carrier-phonon coupling provides the necessary dissipation channel which opens a possibility of excitation transfer driven by the Förster interaction even if the energy mismatch between the dots is much larger than the interaction energy. Phonon-assisted excitation transfer between the quantum states of a molecule was in fact observed in many experiments [5, 35-39]. In closely stacked dots this excitation transfer process is mostly due to phonon-assisted tunneling of carriers. However, for larger separations tunneling is exponentially suppressed. Indeed, phonon-assisted transitions involving tunneling are very inefficient for a $10 \mathrm{~nm}$ separation even though a small energy splitting matches the acoustic phonon energies [39]. In such cases, the transfer is most likely due to the Förster coupling.

This paper presents a theoretical review of a few phenomena that appear in double-dot systems. The existing results, which we originally obtained using various theoretical approaches, are presented within a unified formalism. Moreover, some examples of the interplay between the effects due to the phonon and photon reservoirs are discussed. In Sect. 2 we define the model and our approach to the simulation of its evolution. In Sect. 3 we present a theory of excitation transfer between coupled quantum dots due to interband dipole coupling and carrier-phonon interaction [40]. Next, in Sect. 4 we summarize our studies on collective effects in spontaneous emission [41]. We show that superradiant-like behavior may appear in the optical response of sufficiently strongly coupled pairs of quantum dots even if the two dots are not identical. In addition, we extend the existing result by discussing the effect of phonon-induced transitions on the collective optical properties. In Sect. 5, we discuss the decay of entanglement due to phonon-induced dephasing. Again, we extend the existing study of phonon-related effects on picosecond timescales [42] by discussing the long-time decay due to spontaneous emission and the mutual impact of phonon-related and radiation-related effects. The final Sect. 6 contains concluding remarks and some outlook for a possible further development of the theory.

\section{The system}

The system under study is composed of two coupled QDs with transition energies $\epsilon_{1}$ and $\epsilon_{2}$, interacting with their phonon and photon (radiative) environments. We restrict the discussion to the ground states of excitons in each dot and assume that the spin polarizations of the excitons are fixed. As the exciton dissociation energy in absence of external electric fields is rather large (several to a few tens of $\mathrm{meV}$ ), we consider only spatially direct exciton states, i.e., such that the electron-hole pairs reside in one and the same dot.

Thus, the model includes four basis states $|m n\rangle$, $m, n=0,1$, where $m$ and $n$ denote the number of excitons in the first and second dot, respectively. We will use either the explicit tensor product notation or a contracted one both for the states of the two dots $(|m n\rangle \equiv|m\rangle \otimes|n\rangle)$ and for the operators $\left(|m n\rangle\left\langle m^{\prime} n^{\prime}|\equiv| m\right\rangle\left\langle m^{\prime}|\otimes| n\right\rangle\left\langle n^{\prime}\right|\right)$.

We will describe the evolution in a "rotating basis" defined by the unitary transformation

$$
U=\mathrm{e}^{\mathrm{i} E t(|01\rangle\langle 01|+| 10\rangle\langle 10|+2| 11\rangle\langle 11|) / \hbar},
$$

where $E=\left(\epsilon_{1}+\epsilon_{2}\right) / 2$. The Hamiltonian is then

$$
H=H_{\mathrm{DQD}}+H_{\mathrm{ph}}+H_{\mathrm{rad}}+H_{\mathrm{c}-\mathrm{ph}}+H_{\mathrm{c}-\mathrm{rad}} \text {. }
$$

The first term describes exciton states in the DQD structure

$$
\begin{gathered}
H_{\mathrm{DQD}}=\Delta(|1\rangle\langle 1|\otimes \mathbb{I}-\mathbb{I} \otimes| 1\rangle\langle 1|) \\
+V(|1\rangle\langle 0|\otimes| 0\rangle\langle 1|+\text { H.c. }),
\end{gathered}
$$

where $\Delta=\left(\epsilon_{1}-\epsilon_{2}\right) / 2, \mathbb{I}$ is the unit operator, and $V$ is the coupling between the dots, which can be assumed real. Electron and hole wave functions will be modelled by identical anisotropic Gaussians with identical extensions $l$ in the $x y$ plane and $l_{z}$ along $z$ for both particles,

$$
\psi(\boldsymbol{r}) \sim \exp \left(-\frac{1}{2} \frac{x^{2}+y^{2}}{l^{2}}-\frac{1}{2} \frac{z^{2}}{l_{z}^{2}}\right) .
$$

The coupling $V$ may originate either from the Coulomb (Förster) interaction or from tunnel coupling. In the former case it is related to the distance between the dots and to band structure parameters [40, 41]:

$$
V=\frac{\mathrm{e}^{2}|a|^{2}}{4 \pi \epsilon_{0} \epsilon_{\mathrm{r}} D^{3}} f(D / l) .
$$

Here $e$ is the electron charge, $\epsilon_{0}$ and $\epsilon_{\mathrm{r}}$ are the vacuum 
permittivity and the dielectric constant of the semiconductor, respectively, and

$$
a=\frac{\hbar P_{\mathrm{cv}}}{m_{0} E_{\mathrm{g}}} \approx \frac{\hbar}{\sqrt{2 m_{\mathrm{e}}} E_{\mathrm{g}}},
$$

where $P_{\mathrm{cv}}$ is the interband marix element of the momentum operator, $m_{0}$ and $m_{\mathrm{e}}$ are the free and effective electron masses, and $E_{\mathrm{g}}$ is the band gap. The function $f(x)$ accounts for the correction to the point dipole approximation due to the finite size of the dots (in fact, comparable to $D)[40,43]$ and is given by [44]:

$$
f(x)=\frac{x^{3}}{\sqrt{2 \pi}} \int_{0}^{1} \mathrm{~d} t\left(1-t^{2}\right) \frac{u(t)-x^{2} t^{2}}{u^{5 / 2}(t)} \exp \left(-\frac{x^{2} t^{2}}{2 u(t)}\right),
$$

where $u(t)=1-t^{2}+\left(l_{z} / l\right)^{2} t^{2}$. This correction reduces the coupling for $D \lesssim l$ and removes the $1 / D^{3}$ singularity at $D \rightarrow 0$, while it does not affect the coupling for $D \gg l$ since $f(x) \rightarrow 1$ as $x \rightarrow \infty$. We will neglect the possible biexciton shift (coupling between static, intraband dipole moments).

The phonon modes are described by the free phonon Hamiltonian

$$
H_{\mathrm{ph}}=\sum_{\boldsymbol{k}} \hbar \omega_{\boldsymbol{k}} b_{\boldsymbol{k}}^{\dagger} b_{\boldsymbol{k}}
$$

where $b_{\boldsymbol{k}}, b_{\boldsymbol{k}}^{\dagger}$ are bosonic operators of the phonon modes and $\omega_{k}$ are the corresponding frequencies. Interaction of carriers confined in the DQD with phonons is modelled by the Hamiltonian

$$
\begin{aligned}
& H_{\mathrm{c}-\mathrm{ph}}=(|1\rangle\langle 1| \otimes \mathbb{I}) \sum_{\boldsymbol{k}} f_{\boldsymbol{k}}^{(1)}\left(b_{\boldsymbol{k}}^{\dagger}+b_{-\boldsymbol{k}}\right) \\
& +(\mathbb{I} \otimes|1\rangle\langle 1|) \sum_{\boldsymbol{k}} f_{\boldsymbol{k}}^{(2)}\left(b_{\boldsymbol{k}}^{\dagger}+b_{-\boldsymbol{k}}\right),
\end{aligned}
$$

where $f_{k}^{(1,2)}$ are system-reservoir coupling constants. For Gaussian wave functions, the coupling constants for the deformation potential coupling between confined charges and longitudinal phonon modes have the form $f_{\boldsymbol{k}}^{(1,2)}=$ $f_{\boldsymbol{k}} \mathrm{e}^{ \pm \mathrm{i} k_{z} D / 2}$, where $D$ is the distance between the subsystems and

$$
f_{\boldsymbol{k}}=\left(\sigma_{\mathrm{e}}-\sigma_{\mathrm{h}}\right) \sqrt{\frac{k}{2 \varrho v c_{1}}} \exp \left(-\frac{l_{z}^{2} k_{z}^{2}+l^{2} k_{\perp}^{2}}{4}\right) .
$$

Here $v$ is the normalization volume, $k_{\perp / z}$ are momentum components in the $x y$ plane and along the $z$ axis, $\sigma_{\mathrm{e} / \mathrm{h}}$ are deformation potential constants for electrons/holes, $c_{1}$ is the speed of longitudinal sound, and $\varrho$ is the crystal density.

The third component in our modeling is the radiative reservoir (modes of the electromagnetic field), described by the Hamiltonian

$$
H_{\mathrm{rad}}=\sum_{\boldsymbol{k}, \lambda} \hbar w_{\boldsymbol{k}} c_{\boldsymbol{k}, \lambda}^{\dagger} c_{\boldsymbol{k}, \lambda}
$$

where $c_{\boldsymbol{k}, \lambda}, c_{\boldsymbol{k}, \lambda}^{\dagger}$ are photon creation and annihilation operators and $w_{\boldsymbol{k}}$ are the corresponding frequencies ( $\lambda$ denotes polarizations). The QDs are separated by a distance much smaller than the relevant photon wavelength $\lambda=2 \pi \hbar c / E$, so that the spatial dependence of the EM field may be neglected (the Dicke limit). The Hamiltonian describing the interaction of carriers with the EM modes in the dipole and rotating wave approximations is

$$
H_{\mathrm{c}-\mathrm{rad}}=\Sigma_{-} \sum_{\boldsymbol{k}, \lambda} \mathrm{e}^{-\mathrm{i} E t / \hbar} g_{\boldsymbol{k} \lambda} c_{\boldsymbol{k}, \lambda}^{\dagger}+\text { H.c. },
$$

with

$$
\Sigma_{-}=|0\rangle\langle 1|\otimes \mathbb{I}+\mathbb{I} \otimes| 0\rangle\langle 1|
$$

and

$$
g_{\boldsymbol{k} \lambda}=\mathrm{i} \boldsymbol{d} \cdot \hat{e}_{\lambda}(\boldsymbol{k}) \sqrt{\frac{\hbar w_{\boldsymbol{k}}}{2 \varepsilon_{0} \varepsilon_{\mathrm{r}} v}},
$$

where $\boldsymbol{d}$ is the interband dipole moment $(d=e a)$ and $\hat{e}_{\lambda}(\boldsymbol{k})$ is the unit polarization vector of the photon mode with the wave vector $\boldsymbol{k}$ and polarization $\lambda$. For wide-gap semiconductors with $E \sim 1 \mathrm{eV}$, zero-temperature approximation may be used for the radiation reservoir at any reasonable temperature.

In certain limiting cases, analytical formulae for the evolution of the DQD system may be found. For uncoupled dots $(V=0)$ interacting only with lattice modes (phonons), an exact solution is available [42]. If only the radiative decay is included, a solution in the Markov limit can be obtained [41]. Here, we will use a description which allows one to deal with the simultaneous action of both these environments. We describe the evolution of the reduced density matrix of the DQD system in the interaction picture with respect to $H_{\mathrm{DQD}}$ by the equation

$$
\dot{\rho}=\mathcal{L}_{\text {rad }}[\rho]+\mathcal{L}_{\mathrm{ph}}[\rho] .
$$

Here the first term describes the effect of the radiative decoherence in the Markovian limit in terms of the Lindblad dissipator

$$
\mathcal{L}_{\text {rad }}[\rho]=\Gamma_{\text {rad }}\left[\Sigma_{-}(t) \rho \Sigma_{+}(t)-\frac{1}{2}\left\{\Sigma_{+}(t) \Sigma_{-}(t), \rho\right\}_{+}\right],
$$

where $\Sigma_{-}(t)=\Sigma_{+}^{\dagger}(t)=\mathrm{e}^{\mathrm{i} H_{\mathrm{DQD}} t / \hbar} \Sigma_{-} \mathrm{e}^{-\mathrm{i} H_{\mathrm{DQD}} t / \hbar}$ and

$$
\Gamma_{\mathrm{rad}}=\frac{E^{3}|\boldsymbol{d}|^{2} \sqrt{\epsilon_{\mathrm{r}}}}{3 \pi \epsilon_{0} c^{3} \hbar^{4}}
$$

is the spontaneous decay rate for a single dot. The second term accounts for the interaction with the non-Markovian phonon reservoir. We use the time-convolutionless equation

$$
\begin{aligned}
& \mathcal{L}_{\mathrm{ph}}[\rho]= \\
& -\int_{0}^{t} \mathrm{~d} \tau \operatorname{Tr}_{\mathrm{ph}}\left(H_{\mathrm{c}-\mathrm{ph}}(t),\left(H_{\mathrm{c}-\mathrm{ph}}(\tau), \rho(t) \otimes \rho_{\mathrm{ph}}\right)\right),
\end{aligned}
$$

where $H_{\mathrm{c}-\mathrm{ph}}(t)=\mathrm{e}^{\mathrm{i} H_{\mathrm{DQD}} t / \hbar} H_{\mathrm{c}-\mathrm{ph}} \mathrm{e}^{-\mathrm{i} H_{\mathrm{DQD}} t / \hbar}, \rho_{\mathrm{ph}}$ is the phonon density matrix at the thermal equilibrium, and $\operatorname{Tr}_{\mathrm{ph}}$ denotes partial trace with respect to phonon degrees of freedom.

The above equation of motion for the reduced density matrix strictly reproduces the results in the limiting cases mentioned above. Moreover, for the case of a DQD coupled to phonons with non-vanishing coupling $V$, it yields results reasonably close to those obtained by a much more 
complex correlation expansion technique [40].

In numerical simulations, we take the parameters corresponding to a self-assembled InAs/GaAs system: $\sigma_{\mathrm{e}}-\sigma_{\mathrm{h}}=9 \mathrm{eV}, \rho=5350 \mathrm{~kg} / \mathrm{m}^{3}, c_{\mathrm{l}}=5150 \mathrm{~m} / \mathrm{s}$, the wave function parameters $l=4.5 \mathrm{~nm}, l_{z}=1 \mathrm{~nm}$, and the radiative recombination time (for a single dot) $1 / \Gamma_{\mathrm{rad}}=400 \mathrm{ps}$

\section{Phonon-assisted excitation transfer}

In this section we discuss the evolution of a DQD system coupled only to its phonon reservoir. The system is then described by the Hamiltonian $H=H_{\mathrm{DQD}}+H_{\mathrm{ph}}+$ $H_{\mathrm{c}-\mathrm{ph}}$ [Eqs. (1) and (3)]. We will assume that there is one exciton in the system, initially localized in one of the dots. We will see that the interplay of the coupling and phonon-assisted dissipation leads to irreversible excitation transfer between the dots, which is due to a weak Coulomb (Förster) coupling between them.

In general, if the initial state corresponds to the exciton located in one of the dots (state $|01\rangle$ or $|10\rangle$ ) the evolution is a combination of conservative (unitary) oscillations due to the perturbation induced by the coupling $V$ and a dissipative, irreversible transition towards the lower eigenstate of $H_{\mathrm{DQD}}[45,40]$ induced by the coupling to the phonon continuum. However, in the case of a weak coupling the oscillations are very small and the occupation of the higher-energy dot follows a nearly exponential decay, as shown in Fig. 1. In these computations, the coupling energy $V$ is calculated according to Eq. (2). An interesting feature visible in Fig. 1 is that the rate of the excitation transfer is non-monotonic both in the energy mismatch and in the separation between the dots.
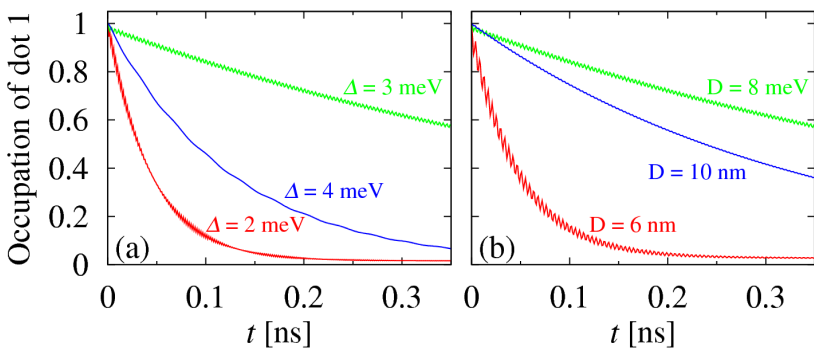

Fig. 1. The occupation of the higher energy QD as a function of time at $T=4 \mathrm{~K}$ : (a) $D=8 \mathrm{~nm}$ and $\Delta$ as shown; (b) $\Delta=3 \mathrm{meV}$ and $D$ as shown.

The nearly exponential decay curve suggests that the process can be described in the Markovian approximation. This is possible, since the typical timescale of the decay process is long compared to the transition frequency $V / \hbar$ between the two single-exciton states. We have shown [40] that the formal long-time limit of Eq. (5) in the rotating wave approximation leads to optical Bloch equations describing the system dynamics in the interaction picture and in the rotating frame related to the eigenstates of $H_{\mathrm{DQD}}$.
In general, upon transforming back to the original basis $|01\rangle,|10\rangle$ and to the Schrödinger picture one obtains a complex evolution. However, in the case of $|V| \ll|\Delta|$, which is of particular practical importance, the eigenstates of $H_{\mathrm{DQD}}$ are very close to $|10\rangle$ and $|01\rangle$. Moreover, in this limit the energy difference between the eigenstates, $\hbar \Omega=2 \sqrt{V^{2}+\Delta^{2}}$ is nearly equal to $2 \Delta$. In this limit, one obtains an exponential excitation transfer with the rate

$$
\Gamma=4 \pi\left(\frac{\tilde{V}}{\Delta}\right)^{2}[R(\Omega)+R(-\Omega)]
$$

with the spectral density

$$
R(\omega)=\frac{2}{\hbar^{2}}\left|n_{\mathrm{B}}(\omega)+1\right| \sum_{\boldsymbol{k}} \sin ^{2} \frac{k_{z} D}{2}\left|f_{\boldsymbol{k}}\right| \delta\left(|\omega|-w_{\boldsymbol{k}}\right) .
$$

Thus, the Markovian equations are particularly useful in the limit of weak coupling, where the Markovian dephasing rate may be identified with the rate of irreversible excitation transfer between the dots.

The rate for the phonon-assisted process is governed, on the one hand, by the amplitude of the Förster coupling which decreases roughly as $1 / D^{3}$. On the other hand, it is strongly influenced by the structure of $R(\Omega)$. The latter oscillates as a function of both $\Omega$ and $D[40]$ due to the interplay between the wavelength of the emitted phonon and the QD separation in the molecule (phonons are preferentially emitted along the strongest confinement limit, i.e., along the DQD axis). In particular, $R(\Omega)$ has a pronounced minimum whenever $\Omega$ is a multiple of $2 \pi \hbar c_{1} / D$ which explains the oscillating dependence on both $\Delta$ and $D$ (see Fig. 2).
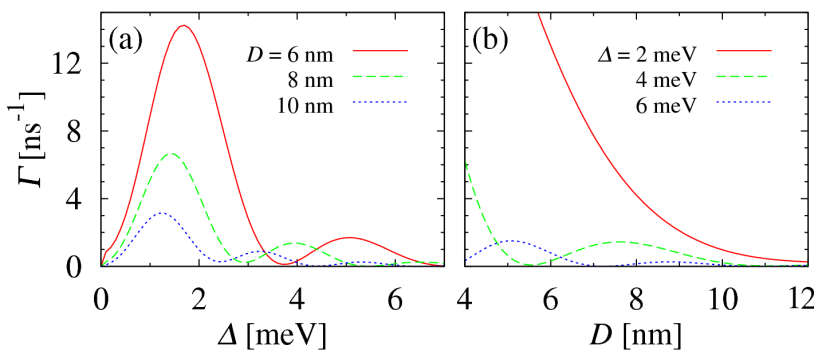

Fig. 2. The rate of phonon-assisted excitation transfer as a function of the energy mismatch for a few values of the QD separation $D$ (a) and as a function of $D$ for a few values of $\Delta(\mathrm{b})$ at $T=4 \mathrm{~K}$.

The transfer rate for $\Delta=4 \mathrm{meV}, D=4.5 \mathrm{~nm}$ is $\Gamma=2.3 \mathrm{~ns}^{-1}$, which is about twice lower than the value of $\Gamma=5.25 \mathrm{~ns}^{-1}$ deduced from fitting to the photon correlation data [5]. It should be noted, however, that our modelling is based on certain choices of parameters that cannot be uniquely determined. First of all, the magnitude of the Förster coupling for a given inter-dot distance can only be roughly estimated, since the value of the parameter $a$ in an inhomogeneous, strained structure is not exactly known. Moreover, the carrier-phonon cou- 
pling constants in the relevant energy (or wave vector) range are strongly geometry dependent. For instance, by changing the localization widths to $l_{\mathrm{e}}=l_{\mathrm{h}}=4.0 \mathrm{~nm}$ and $l_{z}=0.8 \mathrm{~nm}$ one gets a considerably increased value of $\Gamma=3.65 \mathrm{~ns}^{-1}$.

\section{Collective radiative properties of double quantum dots}

In this section, we study the effect of the collective coupling between the two interacting QDs and their electromagnetic environment on the radiative relaxation of carriers. We begin with a model of a DQD interacting only with the EM field. Then, we discuss the effect of the additional phonon-induced relaxation.

In the case of purely electromagnetic environment, the Hamiltonian of the system is $H=H_{\mathrm{DQD}}+H_{\mathrm{rad}}+H_{\mathrm{c}-\mathrm{rad}}$. This Hamiltonian conserves the number of excitations (excitons plus photons). In this case, the solution obtained using Eq. (5) is equivalent to that derived using the Wigner-Weisskopf approach [41].

In Fig. 3 we show the evolution of the average number of excitons in the DQD for two initial states

$$
\left|\psi_{ \pm}(0)\right\rangle=(|01\rangle \pm|10\rangle) / \sqrt{2},
$$

for a DQD with a realistic value of the energy mismatch $\Delta=1 \mathrm{meV}$. For $V \ll \Delta$, both states show simple exponential decay with the rate $\Gamma_{\mathrm{rad}}$. This is understandable, since two different dots emit radiation into different frequency sectors of the reservoir and no collective effects should be expected. The situation changes in the opposite limit, $V \gg \Delta$. Now, one of the states becomes stable (subradiant), while the other (superradiant) state decays exponentially with a twice larger rate. It should be noted that the energy mismatch is much larger than the emission line width, so that the subradiance and superradiance effect is due to the coupling between the dots. In the intermediate range of parameters, the $\mathrm{x}$ exponential. It can be shown [41] that for the superradiant initial state, the number of excitons evolves as

$$
\begin{aligned}
& n(t)=|\boldsymbol{c}(t)|^{2}=\sin ^{2}(\varphi+\pi / 4) \mathrm{e}^{2 \operatorname{Re} \lambda_{-} t} \\
& +\cos ^{2}(\varphi+\pi / 4) \mathrm{e}^{2 \operatorname{Re} \lambda_{+} \mathrm{t}},
\end{aligned}
$$

where

$$
\sin \varphi=\frac{1}{\sqrt{2}}\left(1-\frac{\Delta}{\sqrt{\Delta^{2}+V^{2}}}\right)^{1 / 2}
$$

and

$$
\lambda_{ \pm}=-\frac{\Gamma_{\mathrm{rad}}}{2} \pm \sqrt{-\Delta^{2}+\left(\mathrm{i} V+\Gamma_{\mathrm{rad}} / 2\right)^{2}} .
$$

The values of the two exponents $\lambda_{ \pm}$are shown in the inset of Fig. 3b.

The subradiance and superradiance effects discussed above appear in spite of the large energy mismatch because the single-exciton eigenstates of the DQD Hamiltonian are superpositions of the basis states $|01\rangle$ and $|10\rangle$ which are partly of sub- or superradiant character (for

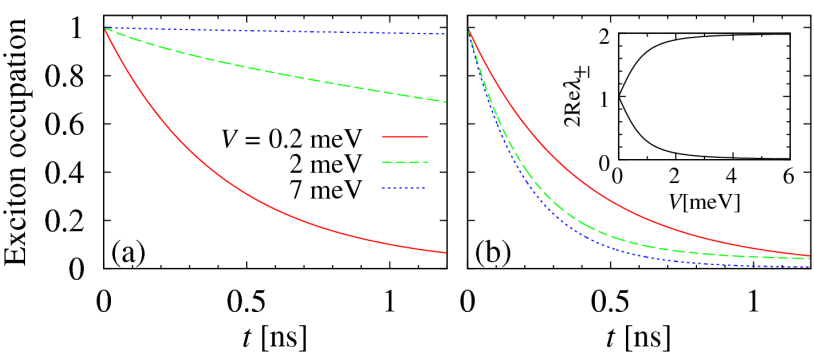

Fig. 3. The exciton occupation for sub- (a) and superradiant (b) states for $\Delta=1 \mathrm{meV}$. The inset in (b) shows the values of the occupation decay rates. Line definitions in (a) refer to both plots.

the -1 and +1 relative phase, respectively). It is therefore clear that the stability of these states is essential for the effect.

When carrier-phonon interaction is included, these states are no longer stable due to excitation transfer processes discussed in Sect. 3. Now, the exciton population decay will depend on the interplay of radiative and phonon-related effects. This is illustrated in Fig. 4, where we plot the exciton number as a function of time for the same two initial states as previously, but now we include the coupling to phonons. Contrary to the purely optical case, the kinetics now depends on the sign of the coupling. For $V<0$, the lowest single-exciton state has a superradiant character. Due to phonon-induced relaxation, the system undergoes transition to this state and the subradiance effect is partly destroyed (see Fig. 4a). An opposite situation takes place for $V>0$ (Fig. 4b). Now, the phonon-induced relaxation consists in a transition to the subradiant state. As a result, the radiative recombination is slowed down by the additional phonon-related decoherence.

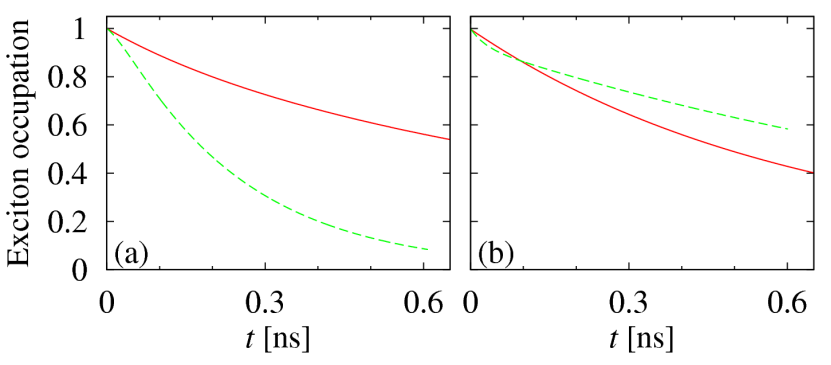

Fig. 4. The exciton occupation for sub- and superradiant states in the presence of phonon-induced relaxation for $\Delta=1 \mathrm{meV}, d=8 \mathrm{~nm}, T=4 \mathrm{~K}$. (a) Subradiant state for $V=-1 \mathrm{meV}$, (b) superradiant state, $V=1 \mathrm{meV}$. Solid lines show the evolution without phonons and the dashed lines with phonons.

Let us note that the superradiant state is particularly relevant for optical experiments since such a bright combination of single exciton states is excited by ultrafast optical pulses from the ground state. Thus, the curves 
plotted in Figs. 3b and 4b directly correspond to the decay of population after an optical excitation.

\section{Entanglement decay}

In this section we discuss the evolution of entanglement between the states of two QDs under dephasing caused by carrier-phonon and carrier-photon interactions. In our earlier work [42] we studied two dots in the absence of excitation transfer coupling, undergoing only phonon-induced pure dephasing. Since that problem was represented by an independent boson model the evolution of the open system could be found in a closed analytical form. We showed that phonon-induced pure dephasing, in spite of its only partial character, can lead to complete decay of entanglement after a finite time. As we pointed out, this happens for certain initial maximally entangled states in which all the coherences are present, that is, all four basis states are involved in the superposition. We studied also the dependence of the entanglement decay on the distance between the dots. We showed that the degree of dephasing-induced disentanglement increases with growing distance between the dots and that non-zero distance is a necessary condition for complete disentanglement. As expected, the degree of dephasing and, therefore, disentanglement increases as the temperature grows. The decay of entanglement due to coupling with the electromagnetic field was also studied [20].

Here, we extend our earlier model [42] by including the radiative decay of the entangled excitons (coupling to the photon reservoir) and excitation transfer interaction $(V \neq 0)$ between the dots. Since the extended model becomes more complicated and presents many new features, we focus on these new elements and restrict the discussion to one initial state and fixed distance $D=8 \mathrm{~nm}$ between the dots. We assume also that the biexciton shift is absent. The dots are assumed different, with the energy mismatch of $\Delta=1 \mathrm{meV}$. In the presence of spontaneous emission and inter-dot coupling, the evolution equation cannot be solved analytically and we use the numerical approach described in Sect. 2. We study the evolution of a maximally entangled "singlet" state

$$
|\psi(t=0)\rangle=\frac{|01\rangle-|10\rangle}{\sqrt{2}} .
$$

As a measure of entanglement, we use the Wootters concurrence $[46,47]$.

The evolution of the entanglement between the dots is shown in Fig. 5. At short (picosecond) timescales, phonon-induced dephasing leads to a drop of concurrence, ending with a temperature-dependent plateau level. In the absence of radiative recombination, the entanglement would remain constant after this initial dephasing stage. However, in the presence of carrierphoton coupling the exciton lifetime becomes finite, which leads to an exponential population decay and, in consequence, to decay of entanglement on the timescales $\sim 1 / \Gamma_{\mathrm{rad}}$.

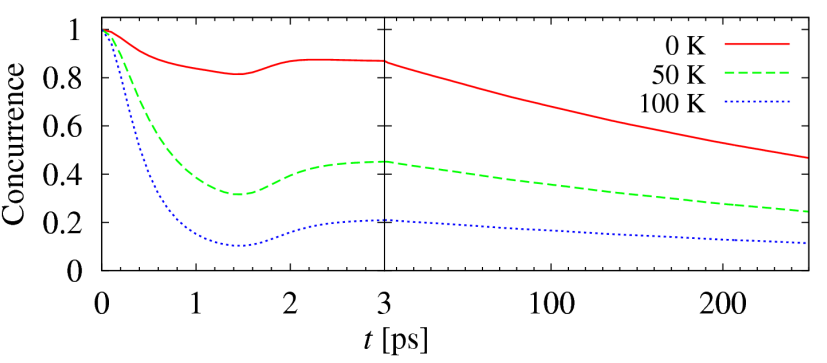

Fig. 5. Evolution of entanglement for uncoupled dots $(V=0)$ at various temperatures.

The situation becomes much more complicated (and more interesting) if the dots are coupled by a transfer-type interaction (that is, $V \neq 0$ ). The evolution in this case is shown in Fig. 6. A few effects can be seen. The most striking feature are the oscillations of concurrence. Since the coupling is comparable to the energy mismatch, the system performs rotations in the single-exciton subspace, coming close to the separable states $|01\rangle$ or $|10\rangle$ every half-period. These oscillations are damped on a timescale of tens of picoseconds, as the excitation is dissipatively transferred to the lower-energy eigenstate of $H_{\mathrm{DQD}}$ by a process discussed above in Sect. 3. Depending on the sign of the interaction, this eigenstate (which can still be entangled) can have either subradiant or superradiant character (for $V>0$ and $V<0$, respectively). This is visible as the difference in the entanglement decay rates between the two cases for long times.

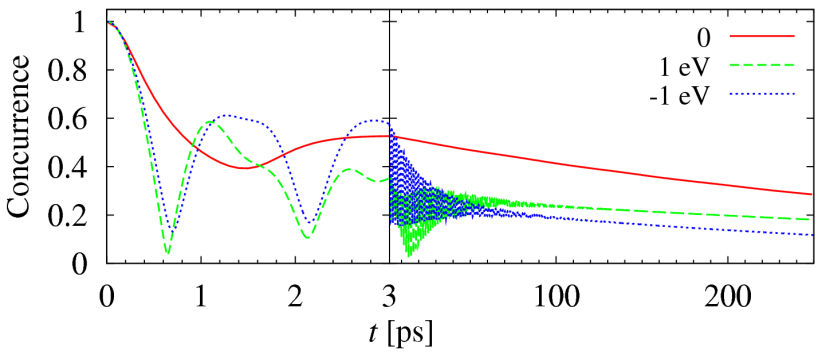

Fig. 6. Evolution of entanglement for coupled dots ( $V= \pm 1 \mathrm{meV}$, as shown) compared to the uncoupled case $(V=0)$ at $T=40 \mathrm{~K}$.

\section{Conclusions and outlook}

In this paper, we have reviewed and extended our recent results on the interaction between systems composed of two quantum dots and their environment. We have shown that collective interaction between the dots and the surrounding radiation reservoir leads to sub- and superradiance effects. In the presence of a coupling between the dots, these collective effects become more stable against the differences between the two transition energies. We have also seen that carrier-phonon coupling leads to new dephasing effects in such double-dot 
systems. In particular, phonon-induced decoherence destroys entanglement between the two dots. This destruction of entanglement is much stronger than local dephasing. We have discussed the joint effect of phonon-induced dephasing and inter-dot coupling. In this case, the most important feature of the system dynamics is the irreversible excitation transfer between the dots.

A new area of interesting and important phenomena emerges if both the spontaneous emission and phonon-induced dephasing are simultaneously taken into account. Most of this field remains to be exploited. As an example of physical effects that may appear due to joint impact of the two reservoirs we have discussed the phonon-induced modification of collective emission due to transitions between subradiant and superradiant states and the interplay of the two dephasing channels in the decay of entanglement between excitons confined in the two dots.

Obviously, the phenomena discussed here are only a fraction of all the physical effects that may be present in a system of two non-identical quantum dots interacting with two different reservoirs (photons and phonons) in the presence of coupling between the dots. In particular, the evolution of double quantum dot systems undergoing simultaneous dephasing via both radiative and phonon-related channel has been studied only in a very limited scope. Investigation of these problems will certainly bring new knowledge not only on the properties of quantum coherence in this specific semiconductor system, but also on general properties of open quantum systems.

\section{Acknowledgments}

This work was supported in parts by the Polish MNiSW (grant No. N N202 105236) and by the Czech Science Foundation (grant No. 202/07/J051).

\section{References}

[1] Q. Xie, A. Madhukar, P. Chen, N.P. Kobayashi, Phys. Rev. Lett. 75, 2542 (1995).

[2] G.S. Solomon, J.A. Trezza, A.F. Marshall, J.S. Harris, Jr., Phys. Rev. Lett. 76, 952 (1996)

[3] B. Gerardot, I. Shtrichman, D. Hebert, P. Petroff, J. Cryst. Growth 252, 44 (2003).

[4] S. Fafard, Z.R. Wasilewski, C.N. Allen, D. Picard, M. Spanner, J.P. McCaffrey, P.G. Piva, Phys. Rev. B 59, 15368 (1999).

[5] B.D. Gerardot, S. Strauf, M.J.A. de Dood, A.M. Bychkov, A. Badolato, K. Hennessy, E.L. Hu, D. Bouwmeester, P.M. Petroff, Phys. Rev. Lett. 95 137403 (2005).

[6] G. Ortner, I. Yugova, G. Baldassarri Höger von Högersthal, A. Larionov, H. Kurtze, D.R. Yakovlev, M. Bayer, S. Fafard, Z. Wasilewski, P. Hawrylak, Y.B. Lyanda-Geller, T.L. Reinecke, A. Babinski, M. Potemski, V.B. Timofeev, A. Forchel, Phys. Rev. B 71, 125335 (2005)
[7] B. Szafran, T. Chwiej, F.M. Peeters, S. Bednarek, J. Adamowski, B. Partoens, Phys. Rev. B 71, 205316 (2005).

[8] B. Szafran, Acta Phys. Pol. A 114, 1013 (2008).

[9] M. Gross, S. Haroche, Phys. Rep. 93, 301 (1982).

[10] N. Skribanowitz, I.P. Herman, J.C. MacGilvray, M.S. Feld, Phys. Rev. Lett. 30, 309 (1973).

[11] M. Scheibner, T. Schmidt, L. Worschech, A. Forchel, G. Bacher, T. Passow, D. Hommel, Nature Phys. 3, 106 (2007).

[12] P. Zanardi, M. Rasetti, Phys. Rev. Lett. 79, 3306 (1997).

[13] P. Zanardi, F. Rossi, Phys. Rev. Lett. 81, 4752 (1998).

[14] P. Borri, W. Langbein, S. Schneider, U. Woggon, R.L. Sellin, D. Ouyang, D. Bimberg, Phys. Rev. Lett. 87, 157401 (2001).

[15] A. Vagov, V.M. Axt, T. Kuhn, W. Langbein, P. Borri, U. Woggon, Phys. Rev. B 70, 201305(R) (2004).

[16] B. Krummheuer, V.M. Axt, T. Kuhn, Phys. Rev. B 65, 195313 (2002)

[17] L. Jacak, P. Machnikowski, J. Krasnyj, P. Zoller, Eur. Phys. J. D 22, 319 (2003).

[18] A. Grodecka, P. Machnikowski, Phys. Rev. B 73, 125306 (2006).

[19] L. Diósi, in: Irreversible Quantum Dynamics, Lecture Notes in Physics, Vol. 622, Eds. F. Benatti, R. Floreanini, Springer, Berlin 2003, p. 157, quant-ph/0301096.

[20] T. Yu, J.H. Eberly, Phys. Rev. Lett. 93, 140404 (2004).

[21] P.J. Dodd, J.J. Halliwell, Phys. Rev. A 69, 052105 (2004).

[22] T. Yu, J.H. Eberly, Phys. Rev. B 68, 165322 (2003).

[23] G.W. Bryant, Phys. Rev. B 47, 1683 (1993).

[24] A. Schliwa, O. Stier, R. Heitz, M. Grundmann, D. Bimberg, Phys. Status Solidi B 224, 405 (2001).

[25] B. Szafran, S. Bednarek, J. Adamowski, Phys. Rev. B 64, 125301 (2001)

[26] M. Bayer, P. Hawrylak, K. Hinzer, S. Fafard, M. Korkusinski, Z.R. Wasilewski, O. Stern, A. Forchel, Science 291, 451 (2001).

[27] G. Ortner, M. Bayer, A. Larionov, V.B. Timofeev, A. Forchel, Y.B. Lyanda-Geller, T.L. Reinecke, P. Hawrylak, S. Fafard, Z. Wasilewski, Phys. Rev. Lett. 90, 086404 (2003).

[28] G. Ortner, M. Bayer, Y. Lyanda-Geller, T.L. Reinecke, A. Kress, J.P. Reithmaier, A. Forchel, Phys. Rev. Lett. 94, 157401 (2005).

[29] H.J. Krenner, M. Sabathil, E.C. Clark, A. Kress, D. Schuh, M. Bichler, G. Abstreiter, J.J. Finley, Phys. Rev. Lett. 94, 057402 (2005).

[30] B.W. Lovett, J.H. Reina, A. Nazir, G.A.D. Briggs, Phys. Rev. B 68, 205319 (2003).

[31] J. Danckwerts, K.J. Ahn, J. Förstner, A. Knorr, Phys. Rev. B 73, 165318 (2006).

[32] T. Förster, Ann. Phys. (Leipzig) 2, 55 (1948).

[33] D.L. Dexter, J. Chem. Phys. 21, 836 (1953).

[34] D.P. DiVincenzo, Fortschr. Phys. 48, 771 (2000). 
[35] R. Heitz, I. Mukhametzhanov, P. Chen, A. Madhukar, Phys. Rev. B 58, R10151 (1998).

[36] A. Tackeuchi, T. Kuroda, K. Mase, Y. Nakata, N. Yokoyama, Phys. Rev. B 62, 1568 (2000).

[37] S. Rodt, V. Turck, R. Heitz, F. Guffarth, R. Engelhardt, U.W. Pohl, M. Straßburg, M. Dworzak, A. Hoffmann, D. Bimberg, Phys. Rev. B 67, 235327 (2003).

[38] G. Ortner, R. Oulton, H. Kurtze, M. Schwab, D.R. Yakovlev, M. Bayer, S. Fafard, Z. Wasilewski, P. Hawrylak, Phys. Rev. B 72, 165353 (2005).

[39] T. Nakaoka, E.C. Clark, H.J. Krenner, M. Sabathil, M. Bichler, Y. Arakawa, G. Abstreiter, J.J. Finley, Phys. Rev. B 74, 121305(R) (2006).

[40] E. Rozbicki, P. Machnikowski, Phys. Rev. Lett. 100, 027401 (2008).
[41] A. Sitek, P. Machnikowski, Phys. Rev. B 75, 035328 (2007).

[42] K. Roszak, P. Machnikowski, Phys. Rev. A $\mathbf{7 3}$, 022313 (2006).

[43] A.O. Govorov, Phys. Rev. B 71, 155323 (2005).

[44] P. Machnikowski, E. Rozbicki, Phys. Status Solidi B 246, 320 (2009).

[45] E. Rozbicki, P. Machnikowski, Acta Phys. Pol. A 112, 197 (2007).

[46] W.K. Wootters, Phys. Rev. Lett. 80, 2245 (1998).

[47] S. Hill, W.K. Wootters, Phys. Rev. Lett. 78, 5022 (1997). 